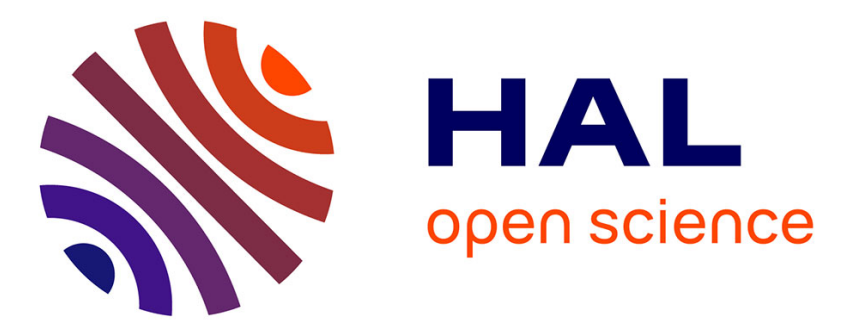

\title{
Littérature, expositions et communication : une conversation
}

Heike Gfrereis, Helmut Neundlinger, Johan Schimanski, Ulrike Spring

\section{To cite this version:}

Heike Gfrereis, Helmut Neundlinger, Johan Schimanski, Ulrike Spring. Littérature, expositions et communication : une conversation. Culture et Musées, 2021, 38, pp.251-272. 10.4000/culturemusees.7194 . hal-03471606

\section{HAL Id: hal-03471606 \\ https://hal.science/hal-03471606}

Submitted on 8 Dec 2021

HAL is a multi-disciplinary open access archive for the deposit and dissemination of scientific research documents, whether they are published or not. The documents may come from teaching and research institutions in France or abroad, or from public or private research centers.
L'archive ouverte pluridisciplinaire HAL, est destinée au dépôt et à la diffusion de documents scientifiques de niveau recherche, publiés ou non, émanant des établissements d'enseignement et de recherche français ou étrangers, des laboratoires publics ou privés. 


\title{
Culture \& Musées
}

Muséologie et recherches sur la culture

$38 \mid 2021$

Patrimonialisations de la littérature

Dossier

\section{Littérature, expositions et communication : une conversation}

Literature, exhibitions and communication: a conversation

Literatura, exposiciones y comunicación: une conversación

\author{
Heike Gfrereis, Helmut Neundlinger, Johan Schimanski eT \\ ULRIKE SPRING \\ Traduction de Dominique Poulot
}

p. $251-272$

https://doi.org/10.4000/culturemusees.7194

\section{Résumés}

Français English Español

Forte de ses compétences en littérature et en théorie et pratique des expositions, Heike Gfrereis est cheffe du département des musées aux Archives de la littérature allemande, à Marbach, et commissaire de nombreuses expositions littéraires. Helmut Neundlinger, conservateur du musée W. H. Auden à Kirchstetten, est un écrivain, chercheur et critique qui travaille au Centre de gestion des collections de musées de l'Université du Danube à Krems, et à la collection littéraire de Basse-Autriche. Leur échange avec deux chercheurs du projet Traum (Transformer les musées d'auteurs) a eu lieu à Oslo en 2019. Nous discutons du désir d'exposer la littérature, de la manière dont on peut se libérer des objets de la manière de désapprendre les notions reçues de la littérature, de l'importance de l'interaction et du jeu, de ce qui peut rendre les auteurs difficiles à exposer et des réalités économiques de l'exposition de la littérature.

Informed by her competence in literature and the theory and practice of exhibitions, Heike Gfrereis is Head of the Museum Department at the Archive of German Literature in Marbach, and curator of many literary exhibitions. Helmut Neundlinger, curator of the W. H. Auden Memorial Museum in Kirchstetten, is a writer, researcher and critic working at the Center for Museum Collections Management at the Danube University Krems and the literary collection of Lower Austria. The following "exchange of knowledge" between them and two researchers in the TRAUM project -Transforming Author Museums- took place in Oslo in 2019. We discuss the desire to exhibit literature and not only biography, how one can free oneself from objects, how to unlearn received notions of literature, the importance of interaction and play, what can make authors difficult to exhibit, and the economic realities of exhibiting literature. 
Gracias a sus conocimientos de literatura y de teoría y práctica de las exposiciones, Heike Gfrereis es directora del Departamento de Museos del Archivo de Literatura Alemana de Marbach y comisaria de numerosas exposiciones literarias. Helmut Neundlinger, conservador del Museo Conmemorativo de W. H. Auden en Kirchstetten, es escritor, investigador y crítico y trabaja en el Centro de Gestión de Colecciones de Museos de la Universidad del Danubio de Krems y en la colección literaria de la Baja Austria. El siguiente "intercambio de conocimientos" entre ellos y dos investigadores del proyecto TRAUM -Transforming Author Museums - tuvo lugar en Oslo en 2019. Hablamos del deseo de exponer literatura y no solo biografía, de cómo uno puede liberarse de los objetos, de cómo desaprender las nociones recibidas de la literatura, de la importancia de la interacción y el juego, de lo que puede hacer que los autores sean difíciles de exponer y de las realidades económicas de la exposición de la literatura.

\section{Entrées d'index}

Mots-clés : expositions littéraires, musées littéraires, maisons d'écrivains, musées d'auteurs, pratiques d'exposition, Auden (W. H.)

Keywords: literary exhibitions, literary museums, writers' houses, author museums, exhibition practices, Auden (W. H.)

Palabras clave: exposiciones literarias, museos literarios, casas de escritores, museos de autores, prácticas expositivas, Auden (W. H.)

\section{Notes de la rédaction}

Traduction de Dominique Poulot revue par Éric Triquet

Cet article est paru à l'origine dans Nordisk Museologi, nº 1, 2020, p. 91-102.

https://journals.uio.no/museolog/article/view/7976

\section{Notes de l'auteur}

Cette conversation a été rendue possible grâce au financement du Conseil de la recherche de Norvège dans le cadre du projet de recherche Traum (Transforming Author Museums) (projet $n^{\circ}$ 251225).

\section{Texte intégral}

\section{Communiquer les auteurs ou la littérature}

Johan : Vous avez tous les deux eu des expériences assez différentes en matière de commissariat d'expositions littéraires. Heike, vous avez travaillé sur des expositions pour des musées et des maisons de la littérature, alors que vous, Helmut, vous avez travaillé dans des maisons d'écrivains. Nous aimerions commencer par vous demander, à partir de ces différentes expériences : quelle est pour vous la facette la plus importante du travail sur une exposition pour un musée ?

2 Heike : J'ai été commissaire d'expositions de littérature depuis plus de vingt ans, avec en tout une centaine d'expositions, petites et grandes, permanentes. Ces expositions n'ont pas eu lieu dans des maisons d'écrivains, mais dans des maisons de la littérature ou des musées, comme le Musée des archives de la littérature allemande à Marbach. Ne pas faire d'expositions dans des maisons d'auteurs signifie avoir affaire à des espaces architecturaux qui ne sont pas liés aux auteurs. La première exposition que j'ai réalisée, avec mes étudiants dans les années 1990, portait sur un auteur souabe, le poète romantique Eduard Mörike (1804-1875). L'exposition s'intitulait Mörike sur l'étagère (Mörike ins Regal gestellt), parce que mon intention était de mettre en scène la littérature - et pas l'auteur : la littérature et le texte. L'exposition s'est tenue dans un musée municipal près de Marbach, à Ludwigsburg, la ville natale d'Eduard Mörike. Marbach abrite le Schiller-Nationalmuseum et le Musée de la littérature moderne, deux musées importants, et les Archives de la littérature allemande, et j'ai réalisé cette exposition avec mes étudiants afin de critiquer les expositions précédentes, car elles présentaient généralement des ensembles, des constellations d'objets mis ensemble afin 
de montrer le « fantôme » de l'auteur. Je lis parce que j'aime la littérature, les textes et la fiction, et non parce que j'aime l'auteur. La littérature est donc pour moi la facette la plus fascinante de toute exposition littéraire.

Helmut : Pour moi, c'est l'inverse ! Je n'ai fait qu'une seule exposition en tant que commissaire principal, à la maison-mémorial W. H. Auden à Kirchstetten, en BasseAutriche, en coopération avec un scénographe très habile, Peter Karlhuber. Nous devions nous occuper de deux choses : l'auteur et l'architecture de la maison. Cela semble très différent de ce que fait Heike, mais je pense que nous avons quelque chose en commun : notre amour de la littérature, des textes. Le fait qu'Auden n'ait pas seulement vécu dans la maison, mais qu'il lui ait aussi consacré l'un de ses plus importants cycles de poèmes (inclus dans un livre sur l'exposition, Neundlinger, 2018) m'a beaucoup aidé. Il a dédié l'ensemble des douze longs poèmes à chaque pièce de la maison. Cela a créé un espace de résonance dans lequel l'exposition a été réalisée.

En même temps, il ne nous reste que deux de ses pièces, car la maison est encore habitée par une famille. Vous avez une vie normale, ordinaire, qui se déroule en bas, et il y a des relations constantes entre l'intérieur et l'extérieur, ou entre ce que nous pourrions appeler l'espace du musée et la vie quotidienne de la communauté. Je décrirais le musée comme une enclave, un enclos. La ville de Kirchstetten se qualifie elle-même de communauté de poètes (Dichtergemeinde), car non seulement le poète anglo-américain W. H. Auden (1907-1973) y a vécu, mais aussi Josef Weinheber (18921945). Ce dernier était un poète autrichien qui a vécu dans la première moitié du xxe siècle et penchait vers le nazisme. Il s'est suicidé en 1945. Auden est venu à Kirchstetten en 1957 et 1958, bien après la mort de Weinheber, mais a écrit un poème sur lui. Il y a donc de nombreux liens avec le contexte sur lesquels on peut s'appuyer dans la littérature d'Auden, pas seulement sur sa vie et sa biographie, et de ce point de vue, on peut dire qu'il y a un rapport entre l'approche de Heike et la nôtre.

5 Ulrike : Un sujet constant dans le projet de recherche Traum était la présence dominante de l'auteur dans les expositions littéraires, non seulement dans les maisons d'auteurs, mais aussi dans les musées littéraires. D’une certaine manière, cela est surprenant, car l'auteur n'est exposé que grâce à ses œuvres. Est-il tellement plus facile d'exposer la biographie et la vie d'un auteur que sa littérature?

$6 \quad$ Heike : Je pense que la convention selon laquelle exposer de la littérature revient à exposer l'auteur découle de la célébration, voire du culte de l'auteur.

7 Johan : Le culte du génie?

8 Heike : Un tel culte implique que le mot écrit de la littérature est quelque chose comme le mot écrit de la Bible. Au xviIIe siècle, la littérature a cessé de faire partie de la vie religieuse, et pour combler ce vide, les gens ont saisi la chose concrète la plus proche de la littérature, et c'était l'écrivain. Je crois que cette convention est encore très dominante, ce qui explique pourquoi tant de visiteurs veulent voir l'auteur et non la littérature, et pourquoi de nombreux organismes de financement et de comités de direction de musées soutiennent également une telle approche biographique. Vous êtes toujours confronté à ce désir et à cet espoir de saisir le fantôme de l'auteur.

9 Ulrike : Ce qui vous unit tous les deux, c'est l'amour de la littérature et des textes. Mais vous travaillez avec des genres muséaux différents : les musées de littérature et la maison d'un auteur. Est-il plus facile de se concentrer sur la littérature dans un musée qui n'est pas associé à la vie de l'auteur ? Dans quelle mesure le contexte détermine-t-il si l'auteur ou son œuvre et la littérature sont au centre de l'exposition?

10 Heike : Ce qui m'intéresse, c'est que dans l'auteur, nous avons une personne qui est assise toute la journée à un bureau et qui se concentre sur l'écriture. Une forme particulière d'habitude de vie est donc associée à ce genre de personne. Rien ne se passe, ce qui est très bon pour l'avancement du travail de l'auteur. C'est fascinant. Un jour, nous avons réalisé une petite exposition avec l'auteur hongrois Péter Nádas (né en 1942), et il voulait qu'elle porte sur la « vie ennuyeuse » des auteurs. Pour moi, cela met en évidence une tension majeure dans les expositions littéraires.

11 Helmut : Dans la tendance d'Auden à se mettre en scène, la réponse à l'ennui est le rituel. On pratique une vie rituelle parce qu'elle est " nue », et on en fait un culte religieux. C'est la raison pour laquelle nous avons affiché le programme de la journée de travail d'Auden sur un mur de l'exposition. Nous ne savons pas s'il s'en tenait 
strictement à cet horaire ou s'il en changeait, mais il a le statut d'une affirmation performative du passé. Il était important pour lui de dire au monde, et même à l'audelà : "Regardez, c'est comme ça que je travaillais, c'était ma façon disciplinée de mener à bien mes affaires. » Pour moi, c'est ici que ça commence à devenir intéressant, parce que la réflexion et la mise en scène de soi se mélangent.

Le théoricien français de la littérature Gérard Genette aurait peut-être appelé l'emploi du temps d'Auden un " paratexte ». Auden a produit un paratexte sur la manière dont il a structuré son œuvre. En tant que spécialiste de la littérature, il est naturel pour moi d'intégrer le document dans l'exposition et de laisser les gens se forger leurs propres idées à ce sujet. Ils pourraient dire : « OK, il dit qu'il se levait à huit heures, et qu'il finissait son travail à six heures avec un whisky, ou un brandy, ou quelque chose de similaire. D'accord, nous pouvons le croire, mais peut-être que ce n'était pas vrai. » Vous créez donc un espace d'imagination autour de l'auteur, ou plutôt, l'auteur crée un espace d'imagination autour de son œuvre. C'est ce que j'appellerais un paratexte.

13 Heike : Et vous créez un espace de significations. C'est la question intéressante : si des objets sont impliqués dans un texte, peuvent-ils fonctionner comme des signes littéraires ? Un verre, par exemple, peut être doté d'une signification en référence au texte, et devenir une forme d'épitexte ou de paratexte. En considérant les objets de cette manière, nous pouvons comprendre d'une part la relation entre le sens et la fiction, d'autre part la réalité d'un texte.

\section{Musées et archives}

Helmut : Heike, vous êtes également très impliquée dans les archives. Pensez-vous que nous traitons ces documents et objets d'une manière différente dans un lieu d'archivage, par rapport à un musée ?

Heike : La manière dont on imagine les archives a toujours à voir avec l'horizon du musée. La succession de l'auteur contient des papiers et des manuscrits. Mes collègues demandent souvent à l'auteur : " Avez-vous quelque chose qui soit très important pour vous ? " Disons, dans le cas de l'auteure autrichienne Barbara Frischmuth (née en 1941), sa houe de jardin. Ce qui se passe ici, c'est que l'auteur dit que c'est significatif, et les archivistes pensent que nous voulons montrer des objets significatifs, et par principe les propres objets favoris des écrivains. Vous ne trouverez donc aucun vestige insignifiant dans les archives.

Mais en tant que conservateur, vous voulez aussi trouver la pièce sans signification. Vous savez qu'elle sera souvent jetée et échappera aux archives, parce que les archives collectent par rapport à la signification des choses, alors que le musée doit combattre ces significations. Dans certains cas, nous sommes donc des rabat-joie, car nous ne jouons pas selon les attentes. Nous montrons les choses sans les investir de sens, voire en déconstruisant leur sens. Nous ne créons pas une aura à partir de cette atmosphère de sens.

Helmut : La collection Auden a été donnée à nos archives, les Archives littéraires de Basse-Autriche, dans les années 1980. À cette époque, le travail d'archivage n'était pas très professionnel. Ils ont pris presque tout, même des choses que l'on ne collectionnerait pas aujourd'hui. Un objet était énorme, et je ne savais même pas comment il s'appelait. Il s'agissait d'un grand verre posé sur une sorte d'étagère de cuisine. Mais ensuite, Peter, mon collègue, le designer, est arrivé et a dit : « Oh, c'est un Weinheber [un siphon à vin]. » Ainsi, avec cet objet, nous avons pu intégrer le poète Weinheber, voisin d'Auden, dans le récit de l'exposition, uniquement parce que, pour une raison quelconque, Auden avait collectionné cet étrange objet. Peter a construit une vitrine spécialement pour cet objet. Le résultat dépend des visiteurs : s'ils savent ce qu'est un Weinheber ou non, mais ceux qui savent ce que c'est et connaissent l'auteur se mettent souvent à rire, car il s'agit d'une déconstruction complète de Josef Weinheber, qui est présenté comme un objet rural. Mais je n'aurais pas découvert cela tout seul et j'ai eu besoin que mon collègue m'en parle. Nous n'avons pas pensé à produire une 
signification particulière ou quelque chose comme ça, nous nous sommes juste amusés avec cet objet.

Heike : Ce travail de conservation va à l'encontre du travail d'archivage qui tente de clarifier les significations et les attributions de manière aussi précise que possible, et qui réduit les ambivalences. Le genre d'exposition qui m'attire réveille ces ambivalences.

\section{La liberté de créer}

Johan : Pourrais-je vous demander, dans le prolongement de la question précédente, quel type d'exposition vous avez réalisé ? Dans un sens, vous êtes très libre. Vous n'êtes pas obligée de suivre la biographie et l'auteur, et vous n'êtes pas obligée d'évoquer la présence d'un auteur. Vous pouvez vous intéresser à de nombreux auteurs, à un motif plutôt qu'à un auteur, à une période ou à une école d'auteurs. Vous pouvez même vous intéresser aux lecteurs plutôt qu'aux auteurs - aux pratiques de lecture.

20 En effet, dans une maison-musée d'écrivain, on pourrait presque être libre d'oublier la biographie. La maison-musée d'un écrivain pose des problèmes très spécifiques quant à la manière de présenter la biographie. La maison d'Auden à Kirchstetten, par exemple, se rapporte principalement à la fin de sa vie, et surtout à la période où il est en Autriche et non à New York. La maison est plus liée au corps et aux habitudes quotidiennes qu'à l'histoire d'une vie. Mais d'un autre côté, Helmut, vous prenez la liberté d'intégrer dans l'exposition à la fois le texte littéraire et l'histoire de sa vie.

Helmut, vous laissez également entendre qu'Auden a façonné sa maison de manière poétique, et que la maison est en fait devenue une partie de la littérature. C'est un point que Harald Hendrix (2008) a soulevé à propos de nombreux auteurs : ils savent que leurs maisons vont devenir des musées, et ils les planifient de cette façon. La question est donc la suivante : que pensez-vous de ces libertés et, lorsque vous travaillez pour une maison d'auteur, comment pouvez-vous prendre certaines libertés et vous libérer d'une perspective purement biographique ?

Heike : Je suis très heureuse de cette liberté, car cela signifie que vous pouvez créer librement, mais il y a aussi un inconvénient en ce qui concerne l'exposition de la littérature, car vous êtes sans corpus. Vous cherchez les objets à exposer selon des critères précis, par exemple lorsque nous ne montrons que des objets qui appartiennent aux Archives de Marbach. Dans d'autres cas, vous êtes libre d'être ouvert à différentes possibilités. Je dois faire une exposition sur Hegel dans les deux semaines à venir et nous n'avons pas d'objets, et vous êtes assis là et vous vous dites : " Pourquoi Hegel ? Quelle œuvre ? Ses Conférences sur l'esthétique ? Oh non, c'est trop grand. La Phénoménologie de l'esprit ? Oh non, c'est trop lourd. » Je serais très heureuse si nous pouvions trouver une boîte verte de manuscrits de Marbach et dire : "Oh, nous allons montrer ça. » Ou bien que nous puissions trouver le bureau de Hegel et l'exposer. Ce manque d'objets est l'inconvénient de cette liberté ${ }^{1}$.

Souvent, nous sommes amenés à faire des expositions en raison de la tradition allemande de célébrer les anniversaires des écrivains. Le paradigme de ces expositions d'auteurs est la grande narration, le grand récit. Il est plus séduisant d'imaginer que les écrivains se concentrent sur une chose en particulier : faire de la littérature. Cela signifie que l'émergence de leur littérature est une révolution permanente de la vie, une machine à expliquer la réalité ou même à l'annihiler. Je trouve cela très excitant : que se passe-t-il quand on fait briller leurs textes dans leurs domaines et que l'on regarde leurs bureaux du point de vue de leurs textes ? Et : que se passe-t-il lorsque vous amenez ces textes dans l'espace et que vous pensez dans l'espace ? Moins on a d'originaux, plus on doit se concentrer sur le corps des textes et le déployer dans les espaces d'exposition. Cela peut donc être un grand avantage d'exposer de la littérature sans archives. D'un autre côté, l'archive est bien sûr aussi un grand moteur herméneutique, car on est constamment confronté à des choses inattendues ou mystérieuses et surtout très concrètes. Les archives donnent à la littérature une réalité et un présent irrévocables. 
Helmut : En tant que visiteur de vos expositions, je dirais que c'est une approche wittgensteinienne, parce que vous encouragez toujours les gens à utiliser les textes ou à utiliser les objets. Il ne s'agit pas toujours d'un texte, parfois c'est une image, ou parfois un objet d'archive, mais votre approche est plus ou moins la même que celle de Wittgenstein qui, dans ses réflexions sur les jeux de langage (Sprachspiele), dit que si vous voulez connaître la valeur d'une chose, vous devez la retourner, vous devez essayer quelque chose avec elle (Wittgenstein, 2009). Je pense que c'est la meilleure approche, car après tout, parcourir une exposition n'est pas exactement la même chose que lire un texte ; c'est plutôt écrire un texte. Depuis un an et demi, vous et moi avons eu des échanges passionnants au cours desquels je vous ai demandé : qui est l'auteur, qui parle dans vos expositions ? Pour moi, vous êtes en quelque sorte un écrivain, un auteur, un poète.

25 Johan, vous avez posé une question sur la puissance d'une maison d'écrivain, et parfois sur la sorte d'enfermement qu'elle illustre. Mon principal avantage est que nous n'avons pas créé une nouvelle exposition mais réécrit une ancienne exposition. Nous avions toujours à l'esprit l'aspect de la salle, la manière dont l'exposition avait fonctionné auparavant, et nous avons essayé de profiter de cette occasion pour répondre à l'exposition telle qu'elle était auparavant. Les conservateurs précédents ont fait ce qu'ils pouvaient en fonction de leur temps et avec les moyens dont ils disposaient. Notre exposition n'est pas une critique de l'ancienne, mais nous avons essayé de jouer avec elle, en la prenant également comme modèle. Nous avons eu la liberté de produire un nouveau récit sur Auden à Kirchstetten.

Ulrike : Si vous deviez transférer l'approche des expositions de Heike dans la maison Auden, est-ce que cela fonctionnerait ou est-ce que cela modifierait ou remettrait en question l'idée du fonctionnement d'une maison d'écrivain ?

Helmut : Je voudrais au moins inviter Heike à faire une petite intervention ou quelque chose comme ça, car il ne s'agit pas vraiment de transférer des choses du passé, mais de créer une méthode à partir de votre objet. Par exemple, ce serait bien de montrer à Heike la voiture délabrée d'Auden, une Coccinelle Volkswagen, et de lui demander : "Comment "lisez-vous" cet objet ? Et comment pourrions-nous relier l'objet à votre lecture ? » C'est ce que j'appellerais la phénoménologie : c'est Husserl (1985) qui a dit que « l'objet est la méthode ». Si l'on prend l'exposition Lachen Cabaret [Cabaret du rire] de Heike, on regarde les choses et on réfléchit à ce que l'on peut en faire, aux objets, à leur matérialité, aux questions qui découlent de l'objet matériel. Si tu venais à Kirchstetten, Heike, tu créerais tes propres idées sur ce que pourrait être la pièce ou même le quartier : comment pourrions-nous étendre Auden à l'ensemble de Kirchstetten ? Que devrions-nous faire avec certains objets ? Par exemple, nous avons un objet très difficile, une voiture - une Coccinelle Volkswagen - qui est en très mauvais état, mais à l'échelle symbolique, c'est l'un des objets les plus importants liés à la maison d'Auden. En effet, c'est l'un des objets les plus fous de la maison, en raison des nombreuses histoires qui l'entourent.

Heike : Je pense qu'il y a un pouvoir fascinant, celui de transformer les choses en signes et vice-versa, de sorte que vous pouvez jouer avec eux et créer votre propre alphabet. On peut montrer des manuscrits comme des objets artificiels. Vous pouvez essayer de trouver la grammaire des choses, et vous pouvez faire de n'importe quoi une chose et de chaque chose un signe, et vice-versa. Et c'est ce qui est fascinant dans la réalisation d'expositions. C'est le problème avec Hegel : nous n'avons pas de choses et seulement quelques textes à Marbach. Nous n'avons pas de manuscrits, pas d'amorces et de traces matérielles de la pensée. Il faut donc entrer dans la pensée par des détours et faire de ses textes imprimés de tels objets initiaux de la pensée. Ou tout simplement inventer de tels objets.

\section{Comment exposer la littérature}

Johan : Mais pour en revenir à cette question de vouloir exposer de la littérature, et peut-être de défier les attentes des gens : ils attendent l'auteur et vous leur donnez de la 
littérature. Comment exposez-vous la littérature?

Je pensais au Musée Balzac, à Paris, la Maison de Balzac, dans ce qui fut son domicile. La pièce dans laquelle l'écrivain français Honoré de Balzac (1799-1850) a écrit est un lieu où la littérature s'est produite - bien que la mode actuelle consistant à exposer, par exemple, la salle de bains de l'auteur (ainsi dans l'appartement d'Ibsen à Oslo) peut également indiquer un lieu où la littérature s'est produite pour l'auteur, puisque les auteurs peuvent s'allonger dans un bain ou aller aux toilettes et penser à leurs prochaines phrases. Dans l'exposition actuelle, la première salle est remplie d'un certain nombre de bustes de Balzac, réalisés par des sculpteurs - et manifestement, la littérature se produisait aussi dans sa tête. Ensuite, il y a une salle avec différentes versions manuscrites du roman sur lequel il travaillait, et c'est aussi un lieu où la littérature se produit - très proche de son corps. Puis il y a une pièce avec de nombreuses images des personnages de ses livres, ce qui est aussi une façon d'exposer la littérature. Et puis il y a des étagères avec ses livres, qui sont aussi de la littérature. Vous voyez aussi des citations de ses textes, qui sont aussi de la littérature.

$\mathrm{Si}$ vous vous rendez à une exposition pour enfants, elle portera également sur la littérature : non pas sur les livres, les textes ou les lieux où ils ont été écrits, mais sur le monde dépeint dans les textes. C'est le cas des musées littéraires consacrés à la littérature pour enfants. De même, la maison elle-même peut faire partie de la littérature, être décrite dans la littérature (comme c'est le cas dans les formes de littérature non fictionnelles, telles que la poésie lyrique).

Comment gérez-vous ces nombreuses manières différentes d'exposer la littérature ?

Heike : Jouer avec les différentes manières de montrer la littérature indique la richesse du genre. Vous pouvez montrer les structures de la littérature, qui sont la première qualité visible d'un texte littéraire. On peut le faire par une lecture attentive. Vous prenez votre crayon dans la main et vous marquez quelque chose avec du rouge, du bleu et du jaune, et alors vous avez une structure très visible et très montrable du texte.

34 En outre, vous avez ce domaine très riche du paratexte et de l'épitexte. Il y a des formes de fantaisie et d'imagination, mais aussi le transfert et la transformation du monde dans le texte.

Helmut : Les exemples que vous mentionnez nous racontent une histoire qui tourne autour de la question de savoir comment exposer la littérature. On pourrait aussi dire qu'on ne peut pas vraiment exposer la littérature, mais qu'on peut exposer la production ou la réception de la littérature. Je ne pense pas qu'il faille choisir une manière spécifique de le faire, car les exemples que vous avez mentionnés s'adressent à différents types de visiteurs : des spécialistes, des enfants, des personnes qui s'intéressent à la biographie des auteurs plutôt qu'à leurs œuvres - ce qui est également légitime.

36 Parfois, le cadre définit ce qui ressort de l'exposition, et parfois on vous laisse plus de liberté. Dans les expositions de Heike, vous êtes toujours agréablement confronté à une approche très créative de la littérature et vous devez trouver votre propre chemin. Une exposition doit convenir à des personnes de tous âges, de six à cent ans. Il s'agit seulement de savoir si quelqu'un est ouvert à la littérature ou à la biographie ; s'il n'est ouvert ni à l'une ni à l'autre, c'est en quelque sorte une perte de temps. Parfois, vous pouvez atteindre des personnes qui ne sont pas très intéressées - c'est une victoire -, mais en général, je pense que le défi est de tirer quelque chose du matériel, au moins.

\section{Jouer avec la littérature}

Ulrike : Nous avons mentionné la littérature pour enfants. Il existe de nombreux parcs à thème qui exposent la littérature en simulant un monde littéraire, et où le visiteur peut interagir avec les protagonistes des œuvres - ils vous parlent littéralement. Citons, par exemple, Moominworld à Naantali, en Finlande (et non le Moominvalley Park à Hannō, au Japon, qui est principalement destiné aux adultes), qui est basé sur les livres Moomintroll de l'auteure finlandaise Tove Jansson (1914-2001), 
ou Astrid Lindgren's World à Vimmerby, en Suède, basé sur divers livres de l'auteure suédoise Astrid Lindgren (1907-2002).

Helmut : On pourrait dire qu'ils reconstituent la littérature.

39 Ulrike : Il est intéressant de constater que l'on accepte d'interagir avec les univers littéraires et leurs protagonistes quand on est enfant, mais qu'on cesse de le faire quand on grandit. Il y a bien l'exemple de la Maison Buddenbrook à Lübeck, qui expose une maison fictive et des scènes de la fiction de l'auteur allemand Thomas Mann (18751955). Mais en général, en vieillissant, on passe de la vie dans la littérature à la littérature, qui devient une partie de notre tête.

40 Heike : Oui, c'est parce que les visiteurs adultes ne sont pas comme les enfants, prêts à se laisser impliquer. Les adultes ont simplement plus d'inhibitions. La maison des Buddenbrook est le décor d'un roman, mais beaucoup de visiteurs pensent que c'est un lieu réel. Ils ne voient pas la différence entre la fiction et la réalité. Je pense que les enfants voient les différences lorsqu'ils interagissent, et qu'ils sont également capables de transformer les mots en signes et en choses. Ils n'ont aucun problème s'ils ne peuvent pas lire une écriture manuscrite, par exemple dans un manuscrit. Les adultes ont un problème. Ils veulent que je leur dise ce que cela signifie, et pourquoi ils devraient lire ceci.

41 Helmut : Les adultes croient à la compétence culturelle quand ils vont à une exposition, et c'est un problème si vous leur donnez un texte ou un objet qu'ils ne peuvent pas décoder tout de suite. Mais je pense aussi que cela vient de la tradition du culte de l'auteur, car si vous êtes une personne adulte, vous avez été à l'école et on vous a dit comment lire la littérature. Elle est considérée comme élitiste - vous pouvez même penser que vous n'êtes pas capable de l'atteindre -, et il devient complètement impossible de jouer avec elle.

Heike : L'enseignement de la littérature dans les écoles n'aborde pas l'ambiguïté, et cela signifie que vous avez en permanence le sentiment de lire les choses de la mauvaise façon. C'est peut-être l'une des raisons pour lesquelles les adultes et les enfants traitent différemment la littérature exposée.

43 Helmut : Oui, ce n'est pas seulement le texte, mais aussi la réception qui est canonisée. C'est quelque chose que nous devons démolir. Nous devrions au moins le déconstruire. Parce qu'alors la littérature a la possibilité de redevenir vivante.

\section{Des musées passifs aux centres interactifs}

Ulrike : En Norvège, il est devenu populaire ces dernières années de créer des centres d'auteurs, ce que nous n'avons pas beaucoup vu dans d'autres pays. L'un des principaux publics, et le principal groupe cible, ce sont les écoliers. L’idée derrière ce genre d'exposition est de s'engager avec la littérature, de la communiquer sous différentes formes, ou d'apprendre de la littérature, mais aussi des auteurs. Ces centres utilisent l'interactivité, les reconstitutions, les jeux, etc., comme principaux outils. Les musées norvégiens ont généralement les écoles comme principal groupe cible, ce qui peut également expliquer la popularité de ces centres littéraires.

Johan : Ces centres ont souvent une architecture moderne, voire une signalétique, qui contribue à l'image de marque du lieu. Ils partagent parfois leurs bâtiments avec des bibliothèques. Ils fonctionnent comme des centres communautaires et, plus généralement, comme des pôles culturels.

Heike : Je connais un cas en Allemagne, le Centre de littérature de Burg Hülshoff près de Münster - lieu de naissance d'Annette Droste-Hülshoff (1797-1848) - qui a tenté quelque chose dans ce sens. Mais en Allemagne, l'approche consiste à organiser des expositions dans des archives ou des bibliothèques. À Marbach, nous voulons trouver un moyen de rendre les musées plus accessibles aux gens et aux élèves, en leur donnant la possibilité d'être interactifs. Les musées ne doivent pas être uniquement des lieux de réception passive. Actuellement, il faut renouveler les expositions un an après 
leur ouverture, car c'est l'événement, et non l'exposition elle-même, qui attire les visiteurs. Je pense donc que nous devons penser davantage comme les centres en Norvège.

Helmut : L'un des objets de notre exposition est un documentaire télévisé sur Auden à Kirchstetten, réalisé en 1967 par la chaîne publique autrichienne ORF dans le cadre d'une série intitulée Le Portrait autrichien - Auden est devenu un Autrichien ! Dans une scène, il se tient debout près de son bureau dans sa salle d'écriture, et nous avons placé notre écran vidéo exactement au même endroit. Il se contente de répondre aux questions et a l'air un peu raide, parce que nous sommes dans les années 1960, et vous entendez la voix de l'intervieweur. Si nous devions un jour produire une version numérique de notre exposition, j'aimerais qu'Auden soit un avatar qui se promène dans le bâtiment et dise : " Je suis W. H. Auden et je suis très heureux que vous me rendiez visite. Entrez et je vais vous montrer ma maison. C'est ici que j'ai écrit mes textes les plus célèbres pendant mes dernières années. Ne regardez pas les bouteilles, j’ai oublié de les emporter », et ainsi de suite. Ce serait l'occasion de réagir à la transformation des habitudes perceptives ; les jeunes n'auraient aucun problème à s'immerger dans un jeu d'avatar d'Auden. Il faudrait bien sûr tenir compte de leurs formes de perception et de narration, mais si vous leur donnez un vieil homme à l'allure très étrange - Auden a dit un jour que son visage ressemblait à un gâteau de mariage abandonné sous la pluie... -, ils seraient intéressés et ils le suivraient, même s'il s'agit de la maison complètement ennuyeuse d'un auteur mort depuis 45 ans.

Johan : La tendance à l'interactivité est le signe d'une démocratisation continue des musées. Bien sûr, les musées littéraires ont toujours été associés à une forme d'accès plus large à l'auteur, allant au-delà de la simple lecture des livres. Aux débuts du culte du génie, on pouvait même visiter la maison de l'auteur vivant. Mais aujourd'hui, il semble que l'on tente de rendre ces musées encore plus accessibles à de nombreux niveaux différents. Cela signifie qu'un grand nombre de nouvelles formes d'expositions et d'expériences apparaissent.

50 Cela signifie-t-il également que nous devons prendre conscience de tout l'éventail de ce que peut être une exposition de littérature ? Par exemple, si vous allez dans une librairie, vous voyez aussi une exposition de littérature, et si vous allez chez quelqu'un, les étagères sont une exposition de littérature. Aujourd'hui, ce ne sont pas seulement les archives, les musées ou les maisons d'auteurs qui organisent des expositions littéraires, mais aussi les bibliothèques locales et nationales. Bien entendu, les bibliothèques exposent souvent la littérature sous la forme de livres, car ce sont les objets qu'elles possèdent dans leurs collections. Y a-t-il un lien entre ce type de démocratisation et de nouvelles formes telles que le genre du centre d'auteur norvégien ? Est-ce une façon de faire de la littérature une expérience plus quotidienne?

Heike : Je pense que la pluralité des pratiques d'exposition littéraire qui n'est pas liée à des institutions spécifiques, le caractère non représentatif de certaines formes d'expositions, pourrait présenter une possibilité. Dans certaines expositions, j'ai l'impression que les conservateurs craignent cette démocratisation. Ils essaient de s'en tenir au culte de l'original, à la fameuse aura des choses, ou ils veulent se cacher derrière un récit objectif. À Marbach, nous devons toujours nous demander s'il est nécessaire de montrer l'original dans tous les cas, en raison des difficultés liées à l'exposition des originaux à la température et à la lumière - les fameux " 50 lux ». L'espace d'exposition est sombre et froid, et pour les visiteurs, il existe peut-être des moyens plus confortables d'apprendre à vivre avec et dans la littérature, de lire des livres peut-être. Les bibliothèques sont des lieux où l'on peut lire, où l'on peut apprendre à lire des livres.

52 Ce matin, j'ai visité le (vieux) Musée Munch à Oslo et j’ai été très surpris. En matière d'exposition, les musées d'art sont à l'opposé des musées de littérature, car ils peuvent montrer l'œuvre d'art - l'objet en soi - aux visiteurs. Dans le cas de la littérature, nous montrons dans la plupart des cas des choses qui font référence à l'imagination. Elles ne sont pas l'objet réel en soi, comme une œuvre d'art. Nos objets ne sont que des objets de substitution. Le Musée Munch, quant à lui, ne se contente pas de montrer les tableaux de Munch, mais présente des similitudes avec une exposition de littérature. Les images sont accrochées au mur, mais en tant que visiteur, vous avez l'impression qu'elles ne 
sont pas l'élément principal. L'essentiel est l'épitexte, le paratexte et l'espace pédagogique du musée. Il est intéressant de noter que l'on ne m'a donné aucune information, mais que le musée est seulement un paysage dans lequel guider les élèves, ce que les visiteurs sont peut-être dans ce cas. Je pense que ce dispositif constitue une forme d'échange entre les musées d'art et les musées de littérature.

Il y a quelques années, le conservateur était la personne qui donnait un sens à l'objet. Je pense qu'aujourd'hui les conservateurs sont des personnes qui rapprochent les gens par le biais d'objets, et qu'ils n'ajoutent plus leurs propres interprétations à une pièce ou à une image.

\section{Littérature exclue}

Johan : Quand on pense démocratie culturelle, il est important de réaliser que nous sommes pris dans une tradition canonique. Nous avons une idée de ce que nous voulons exposer et nous sommes très sélectifs lorsque nous concrétisons cette idée. Nous devons donc nous demander : qu'est-ce qui rend difficile l'exposition d'auteurs et de littérature?

Il s'agit peut-être de savoir comment exposer une littérature controversée. Ce n'est pas tout à fait la même chose que de rester chez soi à la lire, en sécurité dans la sphère privée ; les expositions font bien sûr partie de la sphère publique. Certains auteurs sont devenus discutables. L'exemple le plus célèbre en Norvège est celui de Knut Hamsun (1859-1952) auquel un centre est dédié, le Hamsunsenteret [le Centre Knut Hamsun], près de sa maison d'enfance à Hamarøy. Hamsun s'est affilié au nazisme avant et pendant l'occupation de la Norvège, comme Weinheber l'a fait en Autriche, mais d'une manière différente.

Il y a ensuite la question des auteurs qui ne font pas partie du canon, mais qu'il serait intéressant d'exposer parce qu'ils font précisément partie du champ plus large de la littérature. Je fais ici référence aux auteurs qui ne vivent pas en un seul endroit, qui sont mobiles, les écrivains voyageurs et les auteurs migrants ; aux jeunes auteurs qui ne sont pas encore connus, aux auteurs sans succès ; et aux auteurs dits " mauvais », les auteurs de la littérature populaire. Comment travaillez-vous avec ce type de marginalisation et de difficultés, avec cette absence de communication?

Helmut : Il en va de même pour l'histoire littéraire en général. L'histoire littéraire a toujours produit une liste de noms qui sont blancs, masculins et hétérosexuels. Il est important de déconstruire ce grand récit à propos de l'histoire de la littérature. L'un des aspects les plus intéressants de l'histoire du Musée Auden est qu'il n'existerait pas sans l'engagement de Peter Müller, cofondateur de la Société internationale Auden à la fin des années 1970, et homosexuel lui-même, qui vivait dans le quartier. Il a rendu visite à Auden lorsqu'il avait une vingtaine d'années. Non seulement il vivait dans la campagne autrichienne à la fin des années 1960, mais les activités homosexuelles entre hommes adultes étaient interdites jusqu'en 1971, de sorte qu'il était toujours en danger. Et il y avait cet homme, Auden, originaire d'Amérique ou de Grande-Bretagne, qui vivait là avec son partenaire masculin, Chester Kallman (1921-1975). Ils n'avaient pas de relations sexuelles entre eux, mais leurs amants venaient leur rendre visite. Cette façon de vivre était l'une des principales raisons pour lesquelles ce jeune homme, qui avait également remarqué qu'Auden était un grand poète, voulait sauver son patrimoine autrichien : le bâtiment, les papiers, etc.

Cela me dit bien plus que l'histoire d'un écrivain homosexuel. C'est aussi une histoire sur ce qu'était l'Autriche dans les années 1960 ou 1970, et pourquoi il y avait cette tolérance intéressante envers cet étranger qui était manifestement gay, mais qui chantait aussi dans la chorale de l'église locale. Il s'asseyait dans l'église tous les dimanches, profitant de l'office. La joueuse d'orgue - c'était une très jeune femme à l'époque - vient toujours à nos présentations et nous raconte ce que c'était de jouer de l'orgue à côté d'Auden qui chantait très fort, et parfois pas très correctement.

Cela met en évidence un aspect intéressant de cette forme d'exposition littéraire, car elle vous renseigne sur le patrimoine culturel, sur les situations sociales et politiques, 
sur ce qui est considéré comme un comportement normal et ce qui ne l'est pas, etc. Nous pouvons qualifier les auteurs d'outsiders, aussi je pense que le musée donne le point de vue des outsiders sur la société. Pas seulement avec Auden, évidemment, parce qu'il a toujours été un outsider, il y en a beaucoup d'autres où l'on pourrait trouver des histoires similaires.

Heike : Les expositions littéraires ne sont pas la manière la plus simple de travailler avec la littérature : la lecture est plus facile. En même temps, l'un des grands avantages des expositions littéraires est que vous pouvez réfléchir aux conditions de production et de réception de la littérature.

61 Nous devrions peut-être aussi discuter de l'aspect économique des expositions. Dans le cas des Archives littéraires allemandes, nous devons gagner de l'argent avec nos expositions, en atteignant des objectifs spécifiques. Nous ne devons pas avoir des comptes dans le rouge à la fin de l'année. En même temps, il est très important de ne pas faire seulement de grandes expositions pour atteindre ces objectifs, mais aussi ces expositions plus inconfortables sur les écrivains homosexuels et inconnus. Je pense qu'il est important de faire quelque chose qui va à l'encontre d'un canon et des conventions du genre.

Je voudrais ouvrir le musée à tous, sans frais d'entrée. Après tout, dans le secteur culturel, on ne peut pas calculer comme on le fait dans l'économie de marché.

\section{Bibliographie}

Hendrix (Harald). 2008. «Writers' houses as media of expression and remembrance: from selffashioning to cultural memory ", p. 1-11 in Writers' Houses and the Making of Memory / sous la direction d'Harald Hendrix. New York : Routledge.

Husserl (Edmund). 1985. Die phänomenologische Methode. Ausgewählte Texte I. Édition et introduction de Klaus Held. Stuttgart : Reclam.

Neundlinger (Helmut) (dir.). 2018. Thanksgiving fu" $r$ ein Habitat: W. H. Auden in Kirchstetten. Sankt Pölten : Literaturedition Niederösterreich.

Wittgenstein (Ludwig). 2009. Philosophische Untersuchungen / Philosophical Investigations. Texte allemand avec une traduction anglaise de G. E. M. Anscombe, P. M. S. Hacker et Joachim Schulte ; $4^{\mathrm{e}}$ édition revue par P. M. S. Hacker et Joachim Schulte. Chichester : Wiley-Blackwell.

\section{Notes}

1 Voir : https://www.dla-marbach.de/museen/wechselausstellungen/hegel-und-seine-freunde/ (consulté le 13 septembre 2021).

\section{Pour citer cet article}

Référence papier

Heike Gfrereis, Helmut Neundlinger, Johan Schimanski et Ulrike Spring, « Littérature,

expositions et communication : une conversation », Culture \& Musées, 38 | 2021, 251-272.

Référence électronique

Heike Gfrereis, Helmut Neundlinger, Johan Schimanski et Ulrike Spring, « Littérature,

expositions et communication : une conversation », Culture \& Musées [En ligne], 38 | 2021, mis

en ligne le 10 novembre 2021, consulté le 08 décembre 2021. URL :

http://journals.openedition.org/culturemusees/7194 ; DOI :

https://doi.org/10.4000/culturemusees.7194

\section{Auteurs}

\section{Heike Gfrereis}

Heike Gfrereis est une conservatrice allemande spécialiste de la littérature. Elle dirige depuis vingt ans le département des musées aux Archives de la littérature allemande, à Marbach, et elle a été responsable de très nombreuses expositions. Elle est considérée comme l'une des très grandes commissaires internationales d'expositions littéraires.

Courriel : heike.gfrereis[at]ilw.uni-stuttgart.de 
Helmut Neundlinger

Helmut Neundlinger est un universitaire et auteur qui mène ses recherches sur la littérature et sa patrimonialisation au Centre d'études des collections muséales du département d'art et d'études culturelles de l'Université du Danube à Krems.

Courriel : helmut.neundlinger[at]donau-uni.ac.at

Johan Schimanski

Johan Schimanski est professeur de littérature à l'Université d'Oslo et spécialiste, entre autres, des maisons-musées d'écrivains.

Courriel : johan.schimanski[at]ilos.uio.no

Ulrike Spring

Ulrike Spring enseigne également la littérature à l'université d'Oslo. Le projet de recherche Traum (Transformer les musées d'auteurs) s'intéresse au rôle de la littérature dans le processus démocratique et la construction des identités à une période de changements culturels.

Courriel : ulrike.spring[at]iakh.uio.no

\section{Traducteur}

\section{Dominique Poulot}

\section{Droits d'auteur}

Culture \& Musées 